\title{
THE MAXIMUM $f$-DEPTH SPANNING TREE PROBLEM
}

\author{
JÉRÔME MONNOT
}

LAMSADE, Université Paris-Dauphine, Place du Maréchal de Lattre de Tassigny, 75775 Paris Cedex 16, France.

monnot@lamsade.dauphine.fr

\begin{abstract}
This paper deals with the problem of constructing directed trees of optimal weight and root $r$ with depth at most $f(|V|)$ (called $f-\operatorname{depthDSTP_{r}}$ ). We first prove that the maximization and the minimization versions are equal-approximable under the differential ratio, that measures how the value of an approximate solution is placed in the interval between the worst and the best solutions values of an instance. We show that both problems can be approximately solved, in polynomial time, within differential ratio bounded above by $(\liminf f-1) / \lim \inf f$. Next, we demonstrate that, when dealing with edge distances 1 and 2 , undirected graphs and $f(n)=2$ (called $2-\operatorname{depthST} P_{r}[1,2]$ ), we improve the ratio to $3 / 4$. Based upon these results, we obtain new bounds for standard ratio: a $(\lim \inf f-1) / \lim \inf f$-standard approximation for Max $f$-depthDSTP $P_{r}$ which can be improved to $4 / 5$ for Min $2-\operatorname{depthST} P_{r}[1,2]$ and $7 / 8$ for Max $2-\operatorname{depthST} P_{r}[1,2]$.

Keywords: Approximate algorithms; Differential ratio; Performance ratio; Analysis of Algorithms; Reductions;
\end{abstract}

\section{Introduction}

This paper considers the problem of finding low diameter spanning tree of maximum weight. Given a complete directed graph on $n$ vertices with positive weights on its edges, we look for a directed maximum weight spanning tree with root $r$ and radius bounded by $f(n)$ (the depth depends on the graph order). We refer to this problem as the Max $f-\operatorname{depthDSTP.~To~our~knowledge,~this~problem~has~not~been~}$ studied before, while for any integer function $f$ verifying $\liminf f^{1} \geq 2$ its minimization version has been studied both for directed graphs [25] and undirected graphs [15], [14], [16], [19] (in particular, it is known that the minimization problem is $N P-h a r d)$. We also deal with a variant called $f-\operatorname{depthDSTP}[a ; b]$, where the edge-weights are in the set $\{a, a+1, \ldots, b-1, b\}$.

We focus on the design of approximation algorithms with guaranteed performance ratios, that run within polynomial time and produce sub-optimal solutions. Usually, the scientific community compares the worst-case ratio (here called standard ratio) of the cost of the solution generated by the algorithm to the optimal cost. However, we mainly refer in this article to another ratio called differential ratio which measures the worst ratio of, on the one hand, the difference between the cost of the solution generated by the algorithm and the worst cost, and on the other hand, the difference between the optimal cost and the worst cost. This measure, studied by [3], [12] and more recently by [10], leads to new algorithms taking into account the extreme solutions of the instance, and provides the opportunity to better understand these problems. Besides, we show that there are tight links between both measures for our problems.

The goal of an NPO-optimization problem $\pi=(D, s o l, m, g o a l)^{2}$ with respect to an instance $I$ is to find an optimum solution $x^{*}$ such that $O P T(I)=m\left[I, x^{*}\right]=\operatorname{goal}\{m[I, x]: x \in \operatorname{sol}[I]\}$. Another important solution of $\pi$ is a worst solution $x_{*}$ defined by: $W O R(I)=m\left[I, x_{*}\right]=\overline{\operatorname{goal}}^{3}\{m[I, x]: x \in \operatorname{sol}[I]\}$. In

\footnotetext{
${ }^{1} \lim \inf f$ denotes the quantity $\lim _{p \rightarrow \infty} \inf _{n \geq p} f(n)$

${ }^{2} D$ is the set of instances, sol is the set of feasible solutions, $m$ is the objective function and goal $\in\{$ Max, Min $\}$

${ }^{3}$ If goal $=$ Max, then $\overline{\text { goal }}=$ Min and $\overline{\overline{\text { goal }}}=$ Max.
} 
[3], the term trivial solution referred to as worst solution and all the exposed examples have the property that a worst solution can be trivially computed in polynomial time. For example, this is the case of the maximum Cut problem where, given a graph, the worst solution is the empty edge-set, or the Bin-Packing problem, where we can trivially put the items using a distinct bin per item. On the contrary, since a worst solution of the maximum weight bounded-depth spanning tree is an optimal solution of its corresponding minimization version, the computation of such a solution is far from being trivial! The same property occurs for the Traveling Salesman problem and we have proved that Min TSP and Max TSP (resp. Min TSP[1,2] and Max TSP[1,2]) are 2/3 [23] (resp. 3/4)-differential approximable [24].

1.1. Approximate algorithms and reductions. In order to study algorithm performances, two measures are commonly used: standard ratio [13], [2], [6] and differential ratio [10], [3] and [12].

Definition 1.1. Let $\pi$ be an NPO problem and $x$ be a feasible solution of an instance $I$. We define the performance ratios of $x$ with respect to the instance $I$ as

- (standard ratio) $\rho[\pi](I, x)=\operatorname{Min}\left\{\frac{m[I, x]}{O P T(I)}, \frac{O P T(I)}{m[I, x]}\right\}$

- (differential ratio) $\delta[\pi](I, x)=\frac{W O R(I)-m[I, x]}{W O R(I)-O P T(I)}$

The performance ratio is a number less than or equal to 1 , and is equal to 1 when $m[I, x]=O P T(I)$. Remark that, compared to some definitions, we have inverted the standard performance ratio in the case of minimization problems. Hence, we will always consider the ratio value as being between 0 and 1 . Let $\pi$ be an NPO problem, for any instance $I$ of $\pi$, a polynomial time algorithm $A$ returns a feasible solution $x^{A}$. The performance of $A$ with respect to $R \in\{\delta, \rho\}$ on the instance $I$ is the quantity $R_{A}[\pi](I)=R[\pi]\left(I, x^{A}\right)$. We say that $A$ is an $r$-approximation algorithm with respect to $R$ if for any instance $I$, we have $R_{A}(I) \geq r$.

Definition 1.2. For any performance ratio $R \in\{\delta, \rho\}$,

- an NPO problem belongs to the class $A P X(R)$ if there exists an r-approximation with respect to $R$ for some constant $r \in] 0 ; 1]$.

- an NPO problem belongs to the class $P T A S(R)$ if there exists an r-approximation $A_{r}$, for any constant $r \in] 0 ; 1\left[\right.$. The family $\left\{A_{r}\right\}_{0<r<1}$ is said to be an approximation scheme.

Clearly, the following inclusion holds for any measure $R \in\{\delta, \rho\}: P T A S(R) \subseteq A P X(R)$. As it is usually done, we will denote by $A P X$ and $P T A S$, respectively, the classes $A P X(\rho)$ and $P T A S(\rho)$. As shown in [8], many problems can have different behavior patterns depending on whether the differential or standard ratio is chosen. This is true for Vertex Covering or Dominating Set. Nevertheless, there are some problems that establish some connections between the differential and the standard ratios, like Bin Packing [9] or Traveling Salesman [22], as well as the problems we are dealing with. Consider the following approximation preserving reductions between pairs $(\pi, R)$ :

Definition 1.3. For $\pi_{i} \in N P O$ and $R_{i} \in\{\delta, \rho\}, i=1,2$,

- an $A$-reduction from the pair $\left(\pi_{1}, R_{1}\right)$ to the pair $\left(\pi_{2}, R_{2}\right)$, denoted by $\left(\pi_{1}, R_{1}\right) \leq{ }^{A}\left(\pi_{2}, R_{2}\right)$, is a triplet $(\propto, f, c)$ such that:

(i) $\propto: D_{\pi_{1}} \longmapsto D_{\pi_{2}}$, transforms an instance of $\pi_{1}$ into an instance of $\pi_{2}$ in polynomial time.

(ii) $f: \operatorname{sol}_{\pi_{2}}[\propto(I)] \longmapsto \operatorname{sol}_{\pi_{1}}[I]$, transforms solutions for $\pi_{2}$ into solutions for $\pi_{1}$ in polynomial time.

(iii) $c:[0 ; 1] \longmapsto[0 ; 1]$ (called expansion of the A-reduction) is a function verifying $c^{-1}(0) \subseteq\{0\}$, and $\forall \varepsilon \in[0 ; 1], \forall I \in D_{\pi_{1}}, \forall x \in \operatorname{sol}_{\pi_{2}}[\propto(I)]: R_{2}\left[\pi_{2}\right](\propto(I), x) \geq \varepsilon \Longrightarrow R_{1}\left[\pi_{1}\right](I, f(x)) \geq c(\varepsilon)$

- an $A * P$-reduction from the pair $\left(\pi_{1}, R_{1}\right)$ to the pair $\left(\pi_{2}, R_{2}\right)$, denoted by $\left(\pi_{1}, R_{1}\right) \leq{ }^{A * P}\left(\pi_{2}, R_{2}\right)$, is an $A$-reduction from $\left(\pi_{1}, R_{1}\right)$ to $\left(\pi_{2}, R_{2}\right)$ such that the restriction of function $c$ to an interval $[a ; 1]$ is bijective and $c(1)=1$ (note that $c(\varepsilon)$ does not necessarily verify $c^{-1}(0) \subseteq\{0\}$ ).

If $\left(\pi_{i}, R_{i}\right) \leq^{A * P}\left(\pi_{3-i}, R_{3-i}\right)$ with $c(\varepsilon)=\varepsilon$ for $i=1,2$, we say that $\left(\pi_{1}, R_{1}\right)$ is equivalent to $\left(\pi_{2}, R_{2}\right) . \diamond$ 
The $A$-reduction preserves constant approximation while the $A * P$-reduction preserves approximation schemes. They are natural generalizations of reductions described by the authors of [26], [2] and [7].

The differential ratio measures how the value of an approximate solution is located in the interval between $W O R(I)$ and $O P T(I)$ whereas for a maximization problem, the standard ratio measures how the value of an approximate solution is placed in the interval between 0 and $O P T(I)$. Hence, in this latter case, we have an $A * P$-reduction from the standard ratio to the differential ratio:

Lemma 1.4. If $\pi=(D$, sol, $m, \operatorname{Max}) \in N P O$, then $(\pi, \rho) \leq^{A * P}(\pi, \delta)$ with $c(\varepsilon)=\varepsilon$.

\section{THE $f$-DEPTH CONSTRAINED SPANNING TREE PROBLEM}

The f-depth constrained spanning tree problem, also called the Hop tree problem in [15], [14], [16] or the Shallow-Light spanning tree problem in [25], [19], is formally defined as follow. Let $f$ be an integer function, without loss of generality, we can suppose that $f(n)$ is always an integer between 2 and $n$.

Definition 2.1. Consider a complete directed graph $\overrightarrow{K_{n}}=(V, \vec{E})$ containing a vertex $r \in V$ and nonnegative $\operatorname{costs} d(\vec{e})$ for each directed edge $\vec{e} \in E$. We want to find an optimal-cost directed spanning tree $\vec{T}$ with root $r$ such that the depth of $\vec{T}$ (the maximum number of edges in the unique path from $r$ to any vertex in $\vec{T}$ ) is at most $f(|V|)$, where the cost of a tree is the sum of the weights on its directed edges. We refer this problem as $f-\operatorname{depthDSTP} P_{r}$. When the root $r$ is not specified, we use the notation $f-\operatorname{depthDSTP}$ and when the graph is undirected, we use the notation $f-\operatorname{depthST} P_{r}$. Finally if the function $f$ is constant (i.e., $f(n)=k \forall n$ ) then we use the notation $k$-depthDSTP $P_{r}$.

If goal $=$ Max, the problem is called Max $f-\operatorname{depthDSTP} P_{r}$, else Min $f-\operatorname{depthDSTP}$. We use the notation $f$-depthDSTP $P_{r}$ when we consider without distinction the case goal=Max or goal $=$ Min. $\diamond$

For the undirected case with goal $=$ Min and constant integer function $f$, this problem has been extensively studied in [15], [14], [16] and arises in many applications such as telecommunication network design and facility location. It is $N P$-hard for any $k \geq 2$, even when distances are one and two (see [1] and [20]) and it is generally not in $A P X$. For $k=2$, this problem is $\mathrm{O}(1 / \ln n)$-approximable with the standard ratio and is equivalent to a version of the simple uncapacitated facility location problem whereas its restriction with distances one and two is 4/5-standard approximable and $A P X$-complete [1]. Moreover, it has been proved in [19] that Min $k$-depthSTP $P_{r}$ is $O(1 / \ln n)$-standard approximable for every constant $k \geq 2$ and $\operatorname{Min} f-\operatorname{depthSTP} P_{r}$ is $O\left(1 / n^{\varepsilon}\right)$-standard approximable for any fixed $0<\varepsilon<1$.

Shallow-Light tree introduced and studied in [4] and [18], is a natural generalization of this problem where the diameter is given by another function $l(e)$. Then, we want to find a tree that simultaneously approximate both a minimum spanning tree and a shortest path tree.

For the directed case, the only approximate results are given by a bicriteria approach [21]. An algorithm is said to have a $\left(\rho_{1}, \rho_{2}\right)$-standard approximation if it finds a tree whose weight is at most within a factor $\rho_{1}$ from the optimum for trees of diameter bounded by $k$, but whose diameter is bounded by $\rho_{2} k$ rather than $k$. The main result of [25] suggests a $(2, \ln n)$-standard approximation for Shallow-Light tree problem in directed graphs.

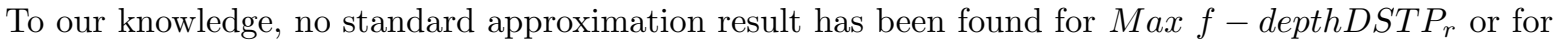
Max $f$-depthSTP (the undirected case), even when the depth does not depend on the graph order.

We show that $M a x f-\operatorname{depthDSTP} P_{r}$ is $(\liminf f-1) / \lim \inf f$-approximable in the differential framework. Then, we deduce the same results for standard ratio.

\section{Some structural PRoperties}

We present some properties of $f-\operatorname{depthDSTP}$ depending on whether the function $f$ is constant or not. We prove that $f-\operatorname{depthDSTP} P_{r}$ is the most general problem to approximate. In other words, 
they are reductions from the undirected or non specific root cases to it. Then, we demonstrate that the maximization, minimization and metric versions are differential equal-approximable. As a second step, we establish some connected relations for the $f-\operatorname{depthDSTP} P_{r}$ family when $f$ is constant.

3.1. Relations between the different subcases. There are simple reductions from $f-\operatorname{depthDSTP}$ and $f-\operatorname{depthSTP} P_{r}$ to $f-\operatorname{depthDSTP} P_{r}$ for both ratios. That is why we will only focus on the problem $f-$ depthDSTP .

Lemma 3.1. for any $R \in\{\rho, \delta\}$, goal $\in\{$ Min, Max $\}$ and arbitrary function $f$, we have:

(i) $($ goal $f$-depthDSTP, $R) \leq{ }^{A * P} \quad\left(\right.$ goal $\left.f-\operatorname{depthDSTP} P_{r}, R\right)$ with $c(\varepsilon)=\varepsilon$.

(ii) $($ goal $f$-depthSTP,$R) \leq^{A * P} \quad\left(\right.$ goal $\left.f-\operatorname{depthDSTP} P_{r}, R\right)$ with $c(\varepsilon)=\varepsilon$.

Proof: We only show the case goal $=$ Max and $R=\delta$.

- For (i): Let $I=\left(\overrightarrow{K_{n}}, d\right)$ be an instance. We define $I_{r}=\left(\overrightarrow{K_{n}}, r, d\right)^{4}$ for all $r \in V$, and if $\overrightarrow{T_{r}}$ is a feasible solution of $I_{r}$, we take $\vec{T}=\operatorname{argmax}\left\{m\left[I, \overrightarrow{T_{r}}\right] \mid r \in V\right\}$. Now, consider $r^{*}$ such that $O P T\left(I_{r^{*}}\right)=O P T(I)$; if $m\left[I_{r^{*}}, \overrightarrow{T_{r^{*}}}\right] \geq \varepsilon O P T\left(I_{r^{*}}\right)+(1-\varepsilon) W O R\left(I_{r^{*}}\right)$, then $m[I, \vec{T}] \geq \varepsilon O P T(I)+(1-\varepsilon) W O R(I)$.

- For (ii): The proof is similar and well known.

Min $f$-depthDSTP $P_{r}$ is not in $A P X$ whereas Max $f-\operatorname{depthDSTP} P_{r}$ is in $A P X$ (as later proved). The asymmetry in the approximability of both versions can be considered as somewhat strange given the structural symmetry existing between them. Since differential approximation is stable under affine

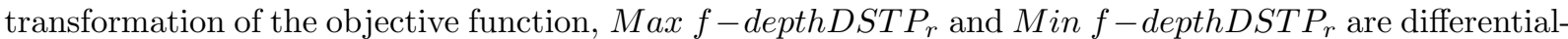

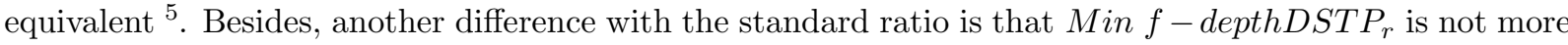
difficult than the same problem with triangular inequality (called metric). Finally, for the restriction where the weight function is bivalued, we can always boil down to the case $a=1$ and $b=2$.

Proposition 3.2. For any integer function $f$, the following assertions hold:

(i) Min $f$-depthDSTP $P_{r}$ is differential-equivalent to Max $f$-depthDSTP $P_{r}$.

(ii) Min $f$-depthDSTP $P_{r}[a, b]^{6}$ is differential-equivalent to Max $f-$ depthDSTP $[a, b]$.

(iii) $f-$ depthDSTP $P_{r}$ is differential-equivalent to metric $f-$ depthDSTP $P_{r}$.

(iv) $f-$ depthDSTP $P_{r}\{a, b\}^{7}$ is differential-equivalent to $f-\operatorname{depthDSTP} P_{r}[1,2]$.

Proof : Let $I=\left(\overrightarrow{K_{n}}, r, d\right)$ be an instance, set $d_{\max }=\max _{\vec{e} \in \vec{E}} d(\vec{e})$ and $d_{\min }=\min _{\vec{e} \in \vec{E}} d(\vec{e})$.

- For (i) and (ii): (Min $\left.f-\operatorname{depthDSTP} P_{r}, \delta\right) \leq{ }^{A * P}\left(\operatorname{Max} f-\operatorname{depthDSTP} P_{r}, \delta\right)$ with $c(\varepsilon)=\varepsilon$.

We transform $I$ into instance $\propto(I)=\left(\overrightarrow{K_{n}}, r, d^{\prime}\right)$ defined by: $\forall \vec{e} \in \vec{E}, d^{\prime}(\vec{e})=d_{\max }+d_{\min }-d(\vec{e})$.

For any feasible solution $\vec{T}$, we have: $m[\propto(I), \vec{T}]=(|V|-1)\left(d_{\text {max }}+d_{\text {min }}\right)-m[I, \vec{T}]$.

Hence if $m[\propto(I), \vec{T}] \geq \varepsilon O P T(\propto(I))+(1-\varepsilon) W O R(\propto(I))$, then $m[I, \vec{T}] \leq \varepsilon O P T(I)+(1-\varepsilon) W O R(I)$. Conversely, the proof is similar since $\propto \circ \propto=I d$.

- For (iii), the proof is similar, except that function d' is now defined by $d^{\prime}(\vec{e})=d_{\max }+d(\vec{e})$.

- Finally, for (iv) the function d' is defined by $d^{\prime}(\vec{e})=1$ iff $d(\vec{e})=a$.

Note that for $f$-depthDSTP , the following easy theorem holds, thus giving a bridge between differential and standard ratios when edge weights belong to an interval $[a, b]$.

Theorem 3.3. For any integer function $f$, we have the following assertion:

\footnotetext{
${ }^{4}$ Note that this is not a reduction according to the definition 1.3 since, for any instance $I$, we are defining several (not one) new instances.

${ }^{5}$ See definition (1.3).

${ }^{6}$ Restriction where all edge weights belong to an interval $[a, b]$.

${ }^{7}$ Restriction where all edge weights belong to a set $\{a, b\}$.
} 
$\left(\right.$ goal $\left.f-\operatorname{depthDSTP} P_{r}[a, b], \rho\right) \leq^{A * P}\left(\right.$ goal $\left.f-\operatorname{depthDSTP} P_{r}[a, b], \delta\right)$ with the expansion verifying:

- $c_{1}(\varepsilon)=\frac{(b-a) \varepsilon}{b_{a}}+\frac{a}{b}$ if goal $=$ Max

- $c_{2}(\varepsilon)=\frac{a}{b-(b-a) \varepsilon}$ if goal $=$ Min

Proof : We only demonstrate the goal $=$ Max case. Let $I$ be an instance and $\vec{T}$ be a feasible solution: if $m[I, \vec{T}] \geq \varepsilon O P T(I)+(1-\varepsilon) W O R(I)$, then $m[I, \vec{T}] \geq c_{1}(\varepsilon) O P T(I)$ since $W O R(I) \geq \frac{a}{b} O P T(I)$

Remark that, with the standard ratio, $f$-depthDSTP $P_{r}[a, b]$ (for $a$ and $b$ not depending on the instance) is "easy" to approximate (i.e., $\left.f-\operatorname{depthDSTP} P_{r}[a, b] \in A P X\right)$ since even a worst solution is an $a / b$ approximation (take $\varepsilon=0$ in theorem (3.3)). Nevertheless, we can deduce from this theorem that Max $f-$ depthSTP $P_{r}[a, b]$ is $A P X-$ complete and the hardness thresholds for standard and differential framework are identical since $\operatorname{Min} f$-depthSTP $P_{r}[a, b]$ is $A P X-$ complete.

Corollary 3.4. If $\liminf f \neq+\infty$ then $f-\operatorname{depthDSTP} P_{r}[a, b] \notin P T A S(\delta)$ unless $P=N P$.

3.2. Relations within the problem family $\operatorname{Max} f-\operatorname{depthDSTP} P_{r}$ when $f$ is constant. These problems share strong structural aspects from the differential point of view according to different values of diameter. We have that Max $2-\operatorname{depthDSTP} P_{r} \in A P X(R)$ if and only if, for any integer $k$, Max $k-$ depthDSTP $P_{r} \in A P X(R), \forall R \in\{\rho, \delta\}$. More specifically, we prove that a "good solution" for Max $k-$

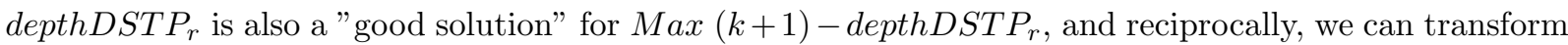
a "good solution" for Max $(k+1)$ - depthDSTP $P_{r}$ into a "good solution" for Max $k-\operatorname{depthDSTP}$.

For a directed spanning tree $\vec{T}$ with root $r$ of $\vec{G}=(V, \vec{E})$, let $p$ be the "father" function of the tree and let $\left(V_{0}, V_{1}, \ldots, V_{q}\right)$ be the partition of $V$ where $V_{i}=\left\{v: r=p^{i}(v)\right\}$ is the $i$-th level vertices subset and $q$ is the depth of $\vec{T}$. So, we have in particular $V_{0}=\{r\}$ and all vertices of $V_{q}$ are leaves of $\vec{T}$. In order to keep it simple, we suppose that $q$ is a multiple of $k$ (we could always add some $V_{i}=\emptyset$ ). Finally, let $\overrightarrow{T_{0}}$ be the directed tree defined by $\vec{T}_{0}=\cup_{v \in V \backslash\{r\}}\{(r, v)\}$, which has a depth equal to 1 . The main idea of the following algorithm consists in discarding some edges of $\vec{T}$ and connecting each resulting tree to the root $r$ in order to obtain a tree with depth $k$.

[shorteningtree $\left.{ }_{k}\right]$

input : A complete directed graph $\overrightarrow{K_{n}}=(V, \vec{E})$ with $r \in V$, edge-valued by $d$ and a directed spanning tree $\vec{T}$ rooted at $r$;

output : 2 directed trees $\operatorname{sol}_{1}$ and $\operatorname{sol}_{2}$ of depth at most $k$ and root $r$;

Build $\left(V_{0}, V_{1}, \ldots, V_{q}\right)$ the partition associated with $\vec{T}$;

For $i=1$ to $k$ do

$$
\begin{aligned}
& \vec{E}_{i}=\vec{T} \backslash\left\{(p(v), v): \exists v \in V_{t}, t \equiv i-1 \bmod k\right\} ; \\
& \text { build } \vec{T}_{i}=\vec{E}_{i} \cup_{j \leq q / k-1} \cup_{v \in V_{j k+i}}\{(r, v)\} ;
\end{aligned}
$$

End for i

$$
\begin{aligned}
& \operatorname{sol}_{1}=\operatorname{argmin}\left\{d\left(\vec{T}_{i}\right), i=1, \ldots, k\right\} ; \\
& \operatorname{sol}_{2}=\operatorname{argmax}\left\{d\left(\vec{T}_{i}\right), i=1, \ldots, k\right\} ;
\end{aligned}
$$

It is easy to see that for any $i \leq k$, the tree $\vec{T}_{i}$ is a feasible solution and that the time-complexity of this algorithm is polynomial with respect to the encoding of the input.

Lemma 3.5. The solutions sol $_{1}$ and sol $_{2}$ produced by [shorteningtree ${ }_{k}$ ] verify:

$$
d\left(\operatorname{sol}_{1}\right) \leq \frac{k-1}{k} d(\vec{T})+\frac{1}{k} d\left(\vec{T}_{0}\right) \leq d\left(\operatorname{sol}_{2}\right)
$$


Proof: We just have to consider the following equality:

$$
\sum_{i \leq k} d\left(\vec{T}_{i}\right)=(k-1) d(\vec{T})+d\left(\vec{T}_{0}\right)
$$

We now establish $A$-reductions with expansion $c(\varepsilon)=\frac{k-1}{k} \varepsilon$. However, these reductions would not have any operational impact since we later prove that $\operatorname{Max} k$-depthDSTP $P_{r}$ is $c(1)$-differential approximable. In other words, the bound resulting from this method is at most $\frac{k-1}{k} \varepsilon<\frac{k-1}{k}$.

Proposition 3.6. The following assertions hold for all $k \geq 2$ and $R \in\{\rho, \delta\}$ :

(i) $\left(\operatorname{Max}(k+1)-\operatorname{depthDSTP} P_{r}, R\right) \leq{ }^{A}\left(\operatorname{Max} k-\operatorname{depthDSTP} P_{r}, R\right)$ with $c(\varepsilon)=\frac{k-1}{k} \varepsilon$

(ii) $\left(\operatorname{Max} k-\operatorname{depthDSTP} P_{r}, R\right) \leq^{A}\left(\operatorname{Max}(k+1)-\operatorname{depthDSTP} P_{r}, R\right)$ with $c(\varepsilon)=\frac{k-1}{k} \varepsilon$

Proof: Let $I=\left(\overrightarrow{K_{n}}, r, d\right)$ be an instance of Max $k$-depthDSTP and denote by $\operatorname{OPT}_{k}(I)$ (resp., $\left.W O R_{k}(I)\right)$ the optimum value (resp., worst value) of $I$. We only demonstrate the differential case.

- For (i): Let $\vec{T}$ be a feasible solution that yields an $\varepsilon$-differential approximation for Max $k-$ $\operatorname{depthDSTP} P_{r}$ (obviously, $\vec{T}$ is a solution for $\operatorname{Max}(k+1)-\operatorname{depthDSTP} P_{r}$ ), we have:

$$
m[I, \vec{T}] \geq \varepsilon O P T_{k}(I)+(1-\varepsilon) W O R_{k}(I)
$$

Thanks to solution $s o l_{2}$ of lemma (3.5) with inputs $\left(I, \vec{T}_{k+1}^{*}\right)$ where $\vec{T}_{k+1}^{*}$ is an optimal solution of $I$ for $\operatorname{Max}(k+1)-\operatorname{depthDSTP} P_{r}$ and $d\left(\vec{T}_{0}\right) \geq W_{O R+1}(I)$, we obtain:

$$
O P T_{k}(I) \geq \frac{k-1}{k} O P T_{k+1}(I)+\frac{1}{k} W O R_{k+1}(I)
$$

Thus, combining (3.2), (3.3) and since $\operatorname{WOR}_{k}(I) \geq W O R_{k+1}(I)$, we obtain that $\vec{T}$ is a $c(\varepsilon)$-differential approximation for $\operatorname{Max}(k+1)-\operatorname{depthDST} P_{r}$.

- For (ii): Let $\vec{T}$ be a feasible solution which yields an $\varepsilon$-differential approximation for Max $(k+1)-$ depthDSTP $_{r}$ and transform $\vec{T}$ into sol $_{2}$ using the polynomial [shorteningtree ${ }_{k}$ ] algorithm.

$$
d\left(\operatorname{sol}_{2}\right) \geq \frac{k-1}{k} m[I, \vec{T}]+\frac{1}{k} d\left(\vec{T}_{0}\right)
$$

Thanks to solution $s_{1}$ of lemma (3.5) with inputs $\left(I, \vec{T}_{k+1}^{-}\right)$where $\vec{T}_{k+1}^{-}$is a worst solution of $I$ for $\operatorname{Max}(k+1)-\operatorname{depthDSTP} P_{r}$, we also have:

$$
\frac{k-1}{k} W_{k+1}(I) \geq \operatorname{WOR}_{k}(I)-\frac{1}{k} d\left(\vec{T}_{0}\right)
$$

Hence, combining (3.4) and (3.5), we obtain the expected result.

Thus, proposition (3.6) leads to the main theoretical result: Max $2-\operatorname{depthDSTP} P_{r} \in A P X(R)$ if and only if, for any function $f, \operatorname{Max} f-\operatorname{depthDSTP} P_{r} \in A P X(R), \forall R \in\{\rho, \delta\}$.

\section{Approximation Results}

We obtain a good solution if we apply [shorteningtree $f$ ] on $\vec{T}^{*}$, an optimal maximum spanning tree of $G$. The complexity time of this algorithm is in $O\left[f(n) \ln \left(d_{\max }\right)(m+n)\right]$ where $m=|\vec{E}|$ and $n=|V|$.

Theorem 4.1. We have the following results:

- $f-\operatorname{depthDSTP} P_{r} \in P T A S(\delta)$ if $\liminf f=+\infty$.

- Else $f$-depthDSTP is differential approximable within $\frac{\liminf f-1}{\liminf f}$ and this ratio is tight. 
Proof: We only demonstrate the second item. Let $I=\left(\overrightarrow{K_{n}}, r, d\right)$ be an instance of Max $f-$ depthDSTP $P_{r}$. We suppose that $n=|V|$ is asymptotically big (else we yield an optimal solution in constant time). Thus, we have $f(n) \geq \liminf f$. The solution $s l_{2}$ of the $\left[\right.$ shorteningtree $\left._{f}\right]$ algorithm with a maximum directed spanning tree $\vec{T}^{*}$ as input leads to the result since $O P T(I) \leq d\left(\vec{T}^{*}\right)$. Let us show that this ratio is tight: consider the following instance $I_{n}=\left(\overrightarrow{K_{n}}, r, d\right)$ such that the sub-graph induced by $V \backslash\{r\}$ is a complete directed graph of $n \lim \inf f$ vertices and the edges-distances are two. Moreover, $d(r, v)=1, \forall v \in V \backslash\{r\}$. So, $\vec{T}^{*}$ is a Hamiltonian path from $r$ to an arbitrary vertex $v$ and:

$$
d\left(\operatorname{sol}_{2}\right)=d\left(\vec{T}_{1}\right)=2 n \liminf f-n, W O R\left(I_{n}\right)=n \liminf f, O P T\left(I_{n}\right)=2 n \liminf f-1
$$

Hence, we obtain $\delta_{\text {shortingtree }_{f}}\left(I_{n}\right) \longrightarrow \frac{\liminf f-1}{\liminf f}$.

We deduce from lemma (1.4) and from theorem (3.3) the two corollaries:

Corollary 4.2. For any integer function $f$ such that $\liminf f<+\infty$ :

- Max $f$-depthDSTP $P_{r}$ is (liminf $\left.f-1\right) / \lim \inf f$-standard approximable

- Max $f$-depthDSTP $P_{r}[a, 2 a]$ is $(2 \lim \inf f-1) / 2 \lim \inf f$-standard approximable.

- Min $f$-depthDSTP $P_{r}[a, 2 a]$ is $\liminf f /(\liminf f+1)$-standard approximable.

Corollary 4.3. For any integer function $f$ such that $\lim \inf f=+\infty$ :

- Max $f$-depthDSTP $P_{r} \in P T A S$

- Min $f-\operatorname{depthDSTP}[a ; 2 a] \in P T A S$

\section{The Depth constraint Minimum Spanning Tree with Distances one and two}

We end this article with studying the special case where the edge-costs one and two, the depth of the

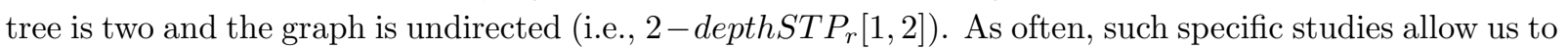
better comprehend the structure and difficulty of those problems (see [27], [11] for the Traveling Salesman problem and [5] for the Steiner Tree problem). For example, we can now suppose that a worst solution is computable in polynomial time though the computation of such a solution for the general problem is $N P-h a r d$ !

We need to introduce some additional notations: $G_{i}, i=1,2$ denotes the sub-graph of $K_{n}$ containing only edges with costs $i$, and $\Gamma_{G}(x)$ is the set of neighbors of $x$ in the graph $G$. For $V^{\prime} \subseteq V$, we write $\Gamma_{G}\left(V^{\prime}\right)=\cup_{x \in V^{\prime}} \Gamma_{G}(x)$. Then, $\Gamma_{G}^{2}(x)=\Gamma_{G} \circ \Gamma_{G}(x)=\cup_{y \in \Gamma_{G}(x)} \Gamma_{G}(y)$.

We present some elementary properties for $\operatorname{Min} 2-\operatorname{depthST} P_{r}[1,2]$ : firstly, we can always suppose that a worst solution is only constituted by edges of cost 2 (otherwise the solution is trivial) and then, it is really the worst valued solution that we can expect. Secondly, we can easily obtain within polynomial time some feasible solutions that contain a lot of edges with cost one:

Lemma 5.1. For an instance $I=\left(K_{n}, r, d\right)$ of Min 2 -depthSTP $P_{r}[1,2]$, the following assertions hold:

(i) We can suppose that $\operatorname{WOR}(I)=2(|V|-1)$.

(ii) We can restrict ourselves to solutions $T$ verifying $d(x, p(x))=1, \forall x \in V \backslash\{r\}, p(x) \neq r$.

(iii) We can restrict ourselves to solutions $T$ verifying $\{(r, x) \mid d(r, x)=1\} \subseteq T$.

Proof : Let $I=\left(K_{n}, r, d\right)$ be an instance of Min $2-\operatorname{depthSTP} P_{r}[1,2]$

- For (i): In fact, we have for every vertex $v_{1} \in V$, the property:

$$
\Gamma_{G_{2}}^{2}\left(v_{1}\right) \cup \Gamma_{G_{2}}\left(v_{1}\right) \neq V \Longrightarrow \Gamma_{G_{1}}^{2}\left(v_{1}\right) \cup \Gamma_{G_{1}}\left(v_{1}\right)=V
$$

Finally we take $v_{1}=r$ and if $W O R(I)<2(|V|-1)$ then we can easily build an optimal tree of cost $n-1$ and depth 2 . 
- For (ii): Let $T$ be a feasible solution and suppose that $d(x, p(x))=2$. Observe that $T^{\prime}=(T \backslash$ $\{(x, p(x))\}) \cup\{(r, x)\}$ is a feasible solution and $d\left(T^{\prime}\right) \leq d(T)$. By iterating the process as much as possible, we obtain the result.

- For (iii), the proof is exactly the same as for (ii).

Observe that this lemma also holds for any integer function $f$. Thanks to lemma (5.1), we show that 2 - depthSTP $P_{r}[1,2]$ is differential reducible to Steiner Dominating Set and since the latter is $3 / 4$ approximable, we obtain a 3/4-differential approximation for $2-\operatorname{depthSTP} P_{r}[1,2]$.

Definition 5.2. Consider a simple graph $G=(V, E)$ and $V_{0} \subseteq V$. The Steiner Dominating Set problem $S D S$ consists in finding a minimum size subset $V^{\prime}$ of vertices containing $V_{0}$ and verifying: $\forall x \notin V^{\prime}, \exists y \in V^{\prime}$ such that $(x, y) \in E$. When $V_{0}=\emptyset$, the problem is called Dominating Set and is denoted by $D S$.

We introduce these new problems because they are very close to $2-\operatorname{depthST} P_{r}[1,2]$ from an differentialapproximability point of view.

Proposition 5.3. We have the following reductions:

(i) $\left(2-\operatorname{depthSTP} P_{r}[1,2], \delta\right) \leq^{A * P}(S D S, \delta)$ with $c(\varepsilon)=\varepsilon$.

(ii) $(D S, \delta) \leq{ }^{A * P}\left(2-\operatorname{depthSTP} P_{r}[1,2], \delta\right)$ with $c(\varepsilon)=\varepsilon$.

Proof : We only demonstrate the case goal = Min.

- For (i): Given $I=\left(K_{n}, r, d\right)$ an instance verifying item(i) of lemma (5.1). We transform $I$ into $\propto(I)=$ $\left(G^{\prime}, V_{0}\right)$ instance of $S D S$ defined by: $V^{\prime}=V \backslash\{r\}, E^{\prime}=\{e: d(e)=1\}$ and $V_{0}=\{v \in V: d(r, v)=1\}$.

Let now $V_{1}$ be a feasible solution of $\propto(I)$ (containing $V_{0}$ ). For every $x$ in $V \backslash V_{1}$, let $e_{x}=(x, y)$ be an edge such that $y \in V_{1}$. We consider $T=\left(\cup_{x \in V_{1}}\{(r, x)\}\right) \cup_{x \notin V_{1}}\left\{e_{x}\right\}$ and we have:

$$
m[I, T]=\left|V^{\prime}\right|-\left|V_{0}\right|+m\left[\propto(I), V_{1}\right]
$$

Let now $T^{*}$ be an optimal solution of $I$ verifying items (ii) and (iii) of lemma (5.1) and we consider the feasible solution $V^{*}=\left\{v:(r, v) \in T^{*}\right\}$; thanks to equation (5.1), we obtain:

$$
O P T(I)=\left|V^{\prime}\right|-\left|V_{0}\right|+O P T(\propto(I))
$$

Since $\operatorname{WOR}(\propto(I))=\left|V^{\prime}\right|$ and $\operatorname{WOR}(I)=2\left|V^{\prime}\right|$ (use item (i) of lemma (5.1)), we have:

$$
\operatorname{WOR}(I) \geq\left|V^{\prime}\right|-\left|V_{0}\right|+W O R(\propto(I))
$$

By combining (5.1),(5.2) and (5.3) we obtain the result.

- For (ii), we transform $I=(V, E)$ into $\propto(I)=\left(K_{n}, r, d\right)$ defined by: the vertex set of $K_{n}$ is $V^{\prime}=V \cup\{r\}$ and $d(e)=1$ iff $e \in E$.

Theorem 5.4. 2 - depthST $P_{r}[1,2]$ is approximable with a differential ratio equal to $\frac{3}{4}$.

Proof : We present an algorithm for $S D S$ which yields a 3/4-differential approximation. We denote $S_{x}=\{x\} \cup \Gamma_{G}(x)$ and $S_{V}^{\prime}=\cup_{x \in V^{\prime}} S(x)$. This algorithm works the following way:

Start with the set $V^{\prime}=V_{0}$ and delete $S_{V_{0}}$ from each $S_{x}$. While there exists a vertex $x_{i} \notin V^{\prime}$ such that $\left|S_{x_{i}}\right| \geq 4$ and $S_{V}^{\prime} \neq V$, add $x_{i}$ to $V^{\prime}$ and delete $S_{x_{i}}$ from each $S_{x}$. Now, any set $S_{x}$ has maximum cardinality 3 and $S_{V^{\prime}} \neq V$. Then, apply Halldorsson's algorithm $L I_{6}$ [17] on the resulting instance and output $V^{\prime}$.

Remark that this algorithm is only a simple transformation of the one given in [17] for the complementary graph coloring problem and the proof is similar. 
We use theorem (3.3) and find the same approximation result of [1] for goal = Min with standard ratio and we obtain a new result for goal $=$ Max.

Corollary 5.5. We have the following results:

- Min 2 - depthST $P_{r}[1,2]$ is approximable with standard ratio equal to $\frac{4}{5}$.

- Max 2 - depthSTP $P_{r}[1,2]$ is approximable with standard ratio equal to $\frac{7}{8}$.

In future research studies, it would be interesting to obtain some non-approximability differential (or standard) results for Max 2 -depthSTP $P_{r}$, similar to those proved in [23] where it is shown that TSP is not approximable with differential ratio greater than $\frac{649}{650}$ unless $P=N P$.

\section{REFERENCES}

[1] L. Alfandari and V. Th. Paschos. Approximating the minimum rooted spanning tree with depth two. Int. Transactions in Oper. Res., 6:607-622, 1999.

[2] G. Ausiello, P. Crescenzi, and M. Protasi. Approximate solutions of NP-optimization problems. Theo. Comp. Sci., 150:1-55, 1995.

[3] G. Ausiello, A. D'Atri, and M. Protasi. Structure preserving reductions among convex optimization problems. J. Comp. Sys. Sci., 21:136-153, 1980.

[4] B. Awerbuch, A. Baratz, and D. Peleg. Cost-sensitive analysis of communication protocols. Proc. 9-th A.C.M. Symp. on Principles of Distributed Computing, pages 177-187, 1990.

[5] M. Bern and P. Plassmann. The Steiner problem with edge lengths 1 and 2. Inf. Proc. Let., 32:171-176, 1989.

[6] P. Crescenzi and V. Kann. A compendium of NP-optimization problems. Available on www address: http://www.nada.kth.se/ viggo/problemlist/compendium.html, 1998.

[7] P. Crescenzi and A. Panconesi. Completness in approximation classes. Inf. and Comp., 93:241-262, 1991.

[8] M. Demange, P. Grisoni, and V. Th. Paschos. Differential approximation algorithms for some combinatorial optimization problems. Theo. Comp. Sci., 209:107-122, 1998.

[9] M. Demange, J. Monnot, and V. Th. Paschos. Bridging gap between standard and differential polynomial approximation: the case of bin-packing. Appl. Math. Let., 12:127-133, 1999.

[10] M. Demange and V. Th. Paschos. On an approximation measure founded on the links between optimization and polynomial approximation theory. Theo. Comp. Sci., 156:117-141, 1996.

[11] L. Engebretsen. An explicit lower bound for TSP with distances one and two. Proc. of 16-th S.T.A.C.S., L.N.C.S. 1563:373-382, 1999.

[12] M. L. Fisher, G. L. Nemhauser, and L. A. Wolsey. An analysis of approximations for finding a maximum weight Hamiltonian circuit. Op. Res., 27(4):799-809, 1979.

[13] M. R. Garey and D. S. Johnson. Computers and intractability. A guide to the theory of NP-completeness. CA. Freeman, 1979.

[14] L. Gouveia. Multicommodity flow models for spanning tree with hop constraints. E.J.O.R., 95:178-190, 1996.

[15] L. Gouveia. Using variable redefinition for computing lower bounds for minimum spanning and Steiner trees with hop constraints. J. Comp., 10(2):180-188, 1998.

[16] L. Gouveia and E. Janssen. Designing reliable tree with two cable technologies. E.J.O.R., 105:552-568, 1998.

[17] M. M. Halldorsson. Approximating k-set cover and complementary graph coloring. Proc. of 5-th I.P.C.O., L.N.C.S. 1084:118-131, 1996.

[18] S. Khuller, B. Raghavachari, and N. Young. Balancing minimum spanning and shortest paths trees. In Proc. 4-th S.O.D.A., pages 243-250, 1993.

[19] G. Kortsarz and D. Peleg. Approximating shallow-light trees. In Proc. 8-th S.O.D.A., pages 103-110, 1997.

[20] P. Manyem and M.F.M Stallmann. Some approximation results in multicasting. Technical report, North Carolina State University, 1996.

[21] M. V. Marathe, R. Ravi, R. Sundaram, S. S. Ravi, D. J. Rosenkrantz, and H. B. Hunt III. Bicriteria network design problems. Proc. 22th Int. Colloq. on Automata Language and Prog., pages 487-498, 1995.

[22] J. Monnot. Families of critical instances and polynomial approximation. PhD thesis, LAMSADE, Université Paris IX, Dauphine, 1998 (in French).

[23] J. Monnot. The maximum Traveling Salesman Problem. submitted article, 2000.

[24] J. Monnot, S. Toulouse, and V. Th. Paschos. Differential approximation results for the traveling salesman problem with distances 1 and 2. submitted article, 2000.

[25] J. S. NAOr and B. Schieber. Improved approximations for shallow-light spanning trees. Proc. F.O.C.S., pages 536-541, 1997. 
[26] P. Orponen and H. Mannila. On approximation preserving reductions: complete problems and robust measures. Technical report, Department of Computer Science, University of Helsinki, 1987.

[27] Ch. Papadimitriou and M. Yannakakis. The traveling salesman problem with distance one and two. Math. Oper. Res., 18:1-11, 1993. 